OPEN ACCESS

Edited by:

David Pacheu-Grau,

University of Zaragoza, Spain

Reviewed by:

Seema Khurana,

University of Houston, United States

lan Jame Holt,

Biodonostia Health Research Institute (IIS Biodonostia), Spain

Eva Carro,

Research Institute Hospital 12 de Octubre, Spain

*Correspondence:

Cristina Ugalde

cugalde@h12o.es

${ }^{\dagger}$ These authors have contributed equally to this work

Specialty section:

This article was submitted to

Cellular Biochemistry,

a section of the journal

Frontiers in Cell and Developmental

Biology

Received: 15 October 2021 Accepted: 03 December 2021

Published: 21 December 2021

Citation:

Illescas M, Peñas A, Arenas J, Martín MA and Ugalde C (2021) Regulation of Mitochondrial Function

by the Actin Cytoskeleton.

Front. Cell Dev. Biol. 9:795838.

doi: $10.3389 /$ fcell.2021.795838

\section{Regulation of Mitochondrial Function by the Actin Cytoskeleton}

\author{
Maria Illescas ${ }^{1 \dagger}$, Ana Peñas ${ }^{1 \dagger}$, Joaquín Arenas ${ }^{1,2}$, Miguel A. Martín ${ }^{1,2}$ and Cristina Ugalde ${ }^{1,2 *}$ \\ ${ }^{1}$ Instituto de Investigación Hospital 12 de Octubre, Madrid, Spain, ${ }^{2}$ Centro de Investigación Biomédica en Red de Enfermedades \\ Raras (CIBERER), Madrid, Spain
}

The regulatory role of actin cytoskeleton on mitochondrial function is a growing research field, but the underlying molecular mechanisms remain poorly understood. Specific actin-binding proteins (ABPs), such as Gelsolin, have also been shown to participate in the pathophysiology of mitochondrial OXPHOS disorders through yet to be defined mechanisms. In this minireview, we will summarize the experimental evidence supporting the fundamental roles of actin cytoskeleton and ABPs on mitochondrial trafficking, dynamics, biogenesis, metabolism and apoptosis, with a particular focus on Gelsolin involvement in mitochondrial disorders. The functional interplay between the actin cytoskeleton, ABPs and mitochondrial membranes for the regulation of cellular homeostasis thus emerges as a new exciting field for future research and therapeutic approaches.

Keywords: mitochondria, actin cytoskeleton, OXPHOS system, gelsolin, mitochondrial disease

\section{INTRODUCTION}

As a major component of the cellular structural network, relevant biological processes like cell division, migration, intracellular transport and organelle organization extensively rely on the dynamics and organization of the actin cytoskeleton. Actin filaments (F-actin) are formed by the polymerization of globular actin monomers (G-actin) in a neat disposition that allows filaments to be polarized. Their remodeling is controlled by a repertoire of actin-binding proteins (ABPs), expressed in a tissue-dependent manner depending on where actin executes cell-specific functions (Lappalainen, 2016; Merino et al., 2020). These proteins regulate a wide spectrum of cellular processes, and are classified regarding their specific action mechanisms: maintenance of the G-actin monomers pool; G-actin nucleation and polymerization of actin filaments and branches; and filaments severing and depolymerization, mainly driven by cofilin and the gelsolin protein superfamily (Silacci et al., 2004).

\section{ACTIN CYTOSKELETON INVOLVEMENT ON MITOCHONDRIAL FUNCTION}

Mitochondria are present in eukaryotic cells and possess a characteristic architecture. The outer mitochondrial membrane (OMM) surrounds the inner mitochondrial membrane (IMM) creating two separate compartments: the internal matrix and intermembrane space (IMS). Mitochondria are fundamental for reactive oxygen species (ROS) production, calcium homeostasis, heat production, cell proliferation or apoptosis (Brookes et al., 2004), and are the main site to important metabolic reactions including the citric acid cycle, amino acids interconversion or $\beta$-oxidation of fatty acids (Nunnari and Suomalainen, 2012), and ATP synthesis through the oxidative phosphorylation (OXPHOS) system (Reid et al., 1966). 


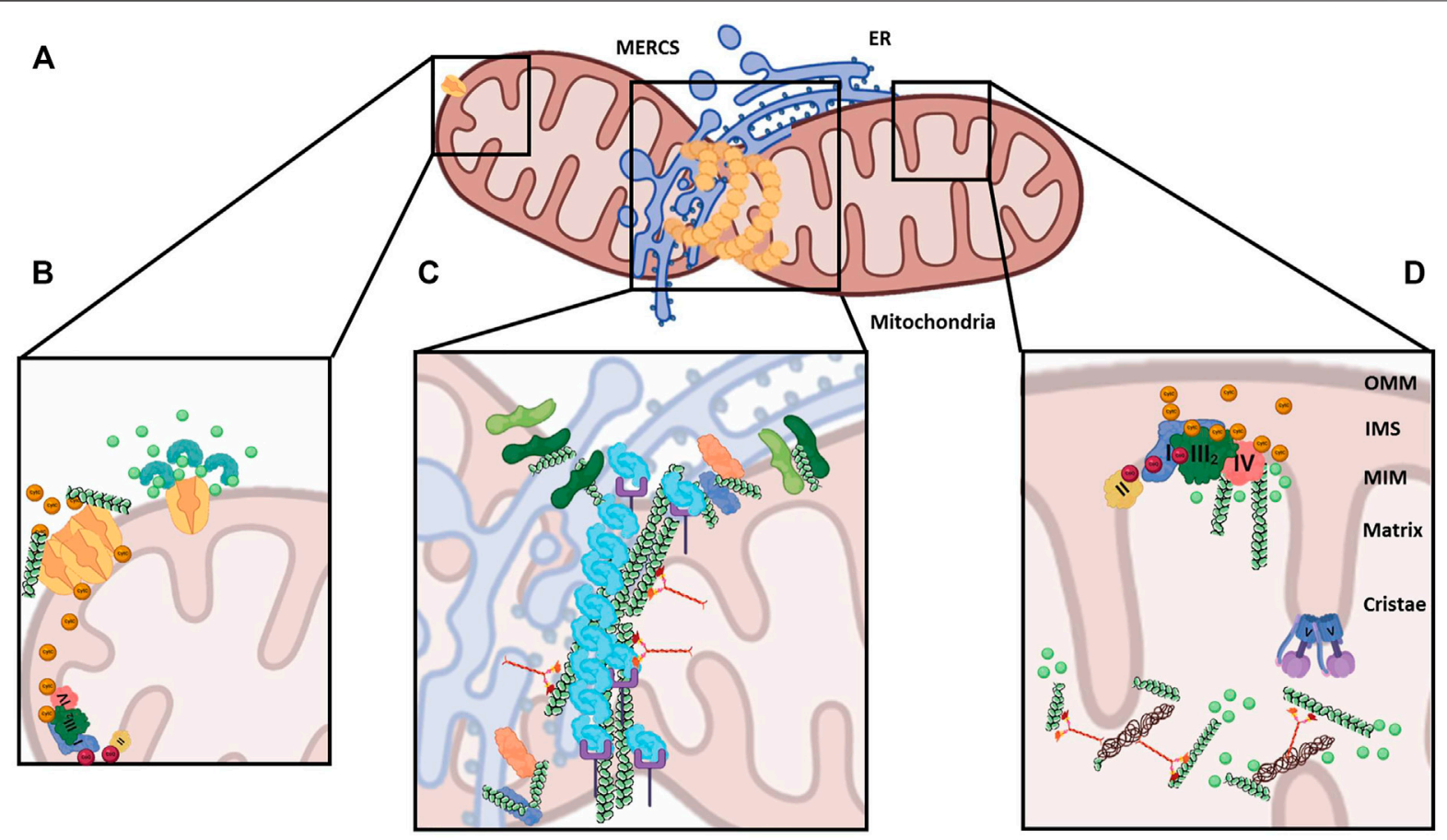

Mitochondrial apoptosis

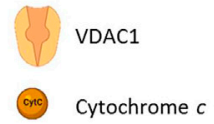

Actin cytoskeleton and ABPs

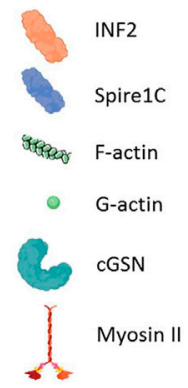

Mitochondrial dynamics

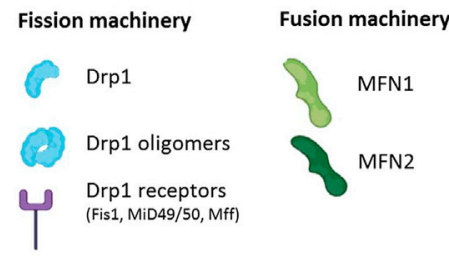

Dynamin

\section{Mitochondrial biogenesis}

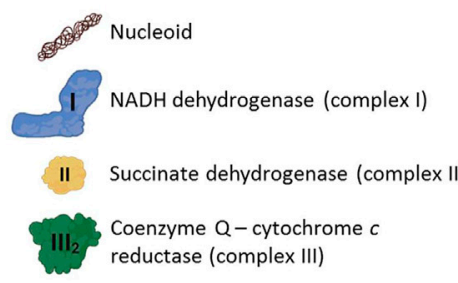

IV Cytochrome coxidase (complex IV)

v ATP synthase (complex V)

Ubiquinone or Coenzyme $Q(\mathrm{CoQ})$

Cytochrome $c$

FIGURE 1 | Regulatory roles of actin cytoskeleton on mitochondrial function. (A) Schematic representation of a mitochondrion interacting with the endoplasmic reticulum (ER) at mitochondrial-endoplasmic reticulum contact sites (MERCS), where dynamin is recruited and fission events take place. (B) Under anti-apoptotic stimuli, actin-binding proteins interact with the voltage-dependent anion channel 1 (VDAC1) in the outer mitochondrial membrane (OMM), preventing actin filaments oligomerization and apoptosis; conversely, actin polymerization favors VDAC oligomerization and induces the apoptotic pathway. (C) Actin cytoskeleton is represented at MERCS, together with ABPs (INF2, Spire1C) and OMM proteins involved in mitochondrial dynamics (MFN1/2). Actin and myosin II localization at MERCSs initiates Drp1 recruitment, which binding to Drp1 receptors (Fis1, MUD49/50, MFF) leads to mitochondrial fission. (D) Actin interacts with mitochondrial respiratory chain (MRC) complexes III and IV in the matrix, controlling the association and dissociation of cytochrome $c$; and together with myosinll, actin regulates the distribution of mitochondrial nucleoids.

Actin is mainly located at the cell membrane, but also at specific mitochondrial subpopulations (Venit et al., 2021). Interactions between mitochondria and the actin cytoskeleton link the essential functions of this organelle to a plethora of cellular physiological processes. Actin filaments primarily modulate mitochondrial dynamics
(Moore et al., 2016; Tilokani et al., 2018), trafficking and autophagy (Kast and Dominguez, 2017), but also mitochondrial biogenesis and metabolism (Fernie et al., 2020). The purpose of this review is to highlight the often overlooked regulatory roles of actin cytoskeleton and ABPs on mitochondrial function. 


\section{Actin Cytoskeleton on Mitochondrial Dynamics}

Mitochondrial function directly depends on its correct morphology and distribution (Scott and Youle, 2010; Sheng, 2014; Chan, 2020), controlled by the balance between fission (division into two or more independent organelles), fusion (formation of a single structure) and mitophagy (clearance of damaged organelles) (Ni et al., 2015). Due to the importance of this system for the maintenance of the cellular metabolic state in mammals (Youle and Van Der Bliek, 2012), the fission and fusion forces require a high degree of regulation by specific molecules along with the actin cytoskeleton. In fact, the dynamic cycling of actin between mitochondrial subpopulations regulates mitochondrial motility and the fission-fusion balance within mitochondrial networks (Moore et al., 2016).

The main role of actin in mitochondrial dynamics is closely linked to the formation of mitochondriaendoplasmic reticulum contacts (MERCs), known as ERMES (ER-mitochondria encounter structure) in yeast (Kornmann and Walter, 2010) (Figure 1A). MERCs are involved in many biological processes like calcium signaling, autophagy, mtDNA replication and phospholipid trafficking (Bononi et al., 2012; Lewis et al., 2016; Xu et al., 2020), besides mitochondrial fission and fusion (Guo et al., 2018; Abrisch et al., 2020). The fusion of adjacent OMMs is orchestrated by mitofusins 1 and 2 (MFN1/2), outer mitochondrial membrane GTPases that form homo- and heterodimers (Friedman et al., 2011). MFN2 is known to tether MERCs, regulating mitochondrial calcium uptake from ER (Han et al., 2021). MERCs are formed before the recruitment of the fission machinery, defining the position of mitochondrial fission sites in a process called ERMD (ERassociated mitochondrial division) (Friedman et al., 2011). The ER-anchored formin INF2 binds to the OMM-located actin nucleator Spire1C, leading to the polymerization of F-actin at MERCs (Korobova et al., 2013; Manor et al., 2015). This leads to the pre-constriction of the OMM driven by the joint action of the ER, actin and non-muscular myosin II. Actin and myosin II ultimately recruit Drp1 (Dynamin Related Protein 1) from the cytosol to the OMM (De Vos et al., 2005), where it oligomerizes (Ji et al., 2015) and interacts with mitochondrial receptors (Mff, MiD49/51 or Fis1), shaping a ring that further constricts and splits the mitochondrion by GTP hydrolysis (Francy et al., 2015). Furthermore, INF2-mediated actin polymerization stimulates the mitochondrial calcium spike, enhancing the metabolic flux by the OXPHOS system and prompting IMM constriction at later stages (Chakrabarti et al., 2018). Interestingly, human cellular models depleted of ABPs, such as spire1C, myosin II or cofilin, showed abnormal mitochondrial motility and morphology, and altered Drp1 recruitment to the OMM (Korobova et al., 2013, 2014; Manor et al., 2015; Pagliuso et al., 2016; Rehklau et al., 2017), pointing to their dual role in OMM pre-constriction and recruitment of fission proteins to the MERCs (Figure 1C).

\section{Actin Cytoskeleton on Mitochondrial Trafficking}

Long-range mitochondrial transport has been primarily studied in neurons (Hirokawa and Takemura, 2005; Schwarz, 2013). This process is mediated by the coupling of mitochondria to microtubule motor proteins (kinesins and dyneins), forming a motor complex together with the OMM GTPase Miro and the adaptor protein Milton/TRAK that mediates mitochondrial transport (van Spronsen et al., 2013; Kruppa and Buss, 2021). Mitochondrial trafficking is closely regulated by intracellular calcium levels, whose increase inhibits both kinesin-dependent anterograde and dynein-dependent retrograde movements (Rintoul et al., 2003); by the PINK1/Parkin pathway, which regulates Miro turnover (Wang et al., 2011; Birsa et al., 2014); or by the nerve growth factor (NGF), responsible for mitochondrial accumulation at the axon region closest to the bead in a manner that involves PI3 kinase and actin (Verburg and Hollenbeck, 2008). The Miro proteins are also key adaptors for the recruitment and stabilization of the ABP myosin19 (Myo19) to the mitochondria (Shneyer et al., 2016; López-Doménech et al., 2018). In human cells, Myo19 has been proposed to regulate the equal segregation of mitochondria to daughter cells during mitosis (Rohn et al., 2014). In yeast, after mitochondrial fission and prior to cell division, mitochondrial populations suffer a poleward movement to both sides of the division plane, exhibiting similar patterns as chromosomes, to contribute to an equitable mitochondrial inheritance to both daughter cells under actin-dependent mechanisms (Boldogh and Pon, 2006; Scott and Youle, 2010). Based on the similarities with cytokinesis, this process was defined as "mitokinesis" (Hatch et al., 2014).

\section{Actin Cytoskeleton on mtDNA Expression and Maintenance}

The mammalian mitochondrial DNA genome (mtDNA) contains 37 genes organized in compact DNA:protein complexes called nucleoids (Clayton, 1991; Spelbrink, 2010), whose expression requires a high degree of coordination with the nuclear genome (Rampelt and Pfanner, 2016). In yeast, the ERMES complex regulates the stability and organization of mtDNA in nucleoids in an actin-dependent manner (Boldogh and Pon, 2006); in mammals, MERCs are spatially-linked to mitochondrial nucleoids, regulating their distribution, division and active transportation by the microtubules (Lewis et al., 2016). Although the presence of actin inside mitochondria and its functional connection with mtDNA was debated for decades (Venit et al., 2021), recent super-resolution microscopy-based studies probed the presence of $\beta$-actin-containing structures inside the mitochondrial matrix (Dadsena et al., 2021). Moreover, human cells lacking $\beta$-actin showed higher sensitivity to stress caused by loss of mitochondrial membrane potential $(\Delta \Psi)$ plus perturbed mtDNA mass and nucleoid organization (Xie et al., 2018), suggesting a regulatory role in mtDNA transcription and quality control. Besides actin, myosin II is also associated with purified mitochondrial nucleoids, and its 
silencing produces mtDNA abnormalities (Reyes et al., 2011). These evidences support the role of actin and ABPs in mitochondrial nucleoid segregation and mtDNA transcription and maintenance, likely through formation of a "mitoskeleton" network supporting mtDNA inheritance (Figure 1D).

\section{Actin Cytoskeleton on Mitochondrial Metabolism}

Actin filaments are indispensable for the full activation of metabolic pathways that subsequently regulate mitochondrial function. For instance, activation of glycolytic enzymes such as aldolase or glyceraldehyde phosphate dehydrogenase, may occur through their direct binding to F-actin (Arnold and Pette, 1968). Aldolase is trapped by the actin cytoskeleton, whose release under PI3K activation increases aldolase activity, thus enhancing glycolysis (Hu et al., 2016). F-actin bundles may also sequester TRIM21 (Tripartite Motif-containing Protein 21), reducing the access of this E3 ubiquitin ligase to its substrates, such as the ratelimiting metabolic enzyme phosphofructokinase (PFK), thus maintaining high glycolytic rates (Park et al., 2020).

Interestingly, in brain mitochondria actin regulates the retention of cytochrome $c$ between respiratory chain complexes III and IV by its direct association with both complexes, and inhibition of actin polymerization with cytochalasin $b$ enhanced mitochondrial respiration through increased complex IV activity (Takahashi et al., 2018) (Figure 1D).

\section{Actin Cytoskeleton on Apoptosis}

Mitochondria represent a major component in the cellular apoptotic machinery (Dadsena et al., 2021), influencing relevant processes like development, cell turnover or immune responses (Man, 2016). During the intrinsic pathway, increased OMM permeabilization and cristae disorganization initiate the release of pro-apoptotic factors (such as cytochrome $c$ or AIF) from the IMS into the cytosol, prompting the formation of the apoptosome protein complex that activates caspases and subsequent chromatin fragmentation (Portt et al., 2011). Actin itself is a substrate of caspase-mediated cleavage (Kayalar et al., 1996), leading to a $15-\mathrm{kDa}$ fragment that can be N-myristoylated and targeted to mitochondria (Utsumi et al., 2003). This interaction modulates ROS production through the regulation of OMM permeabilization by opening-closing membrane channels. In yeast, monomeric actin interacts with the voltagedependent anion channel (VDAC), thus impacting on apoptosis modulation via interfering with the exchange of metabolites and energy between mitochondria and the cytosol (Xu et al., 2001; Roman et al., 2006). Disruption of actin dynamics causes a dramatic loss of mitochondrial $\Delta \Psi$, increased ROS and cell death (Gourlay and Ayscough, 2005). Studies in human cell lines treated with actin-disrupting drugs, affecting both actin stabilization and depolymerization, also reinforced the requirement of actin remodeling for the induction of the intrinsic apoptosis pathway (Odaka et al., 2000; Yamazaki et al., 2000) (Figure 1B).
Many ABPs are actively involved in apoptosis regulation in both yeast and mammals (Franklin-Tong and Gourlay, 2008). One well-studied ABP participating in this process is cofilin. During apoptosis induction in mammalian cells cofilin loses its actin-binding affinity, being translocated to the mitochondria prior to the permeability transition pore (mPTP) opening that promotes cytochrome $c$ release and apoptosis progression (Chua et al., 2003; Roh et al., 2013). This process could be mediated by the interaction of cofilin with Drp1 (Hu et al., 2020), although the binding of cofilin to G-actin seems enough to induce its mitochondrial translocation (Rehklau et al., 2012). Under oxidative stress, oxidized cofilin is also translocated to the mitochondria, promoting mitochondrial fission and triggering the release of cytochrome $c$ leading to apoptosis (Klamt et al., 2009; Lapeña-Luzón et al., 2021). Other relevant ABPs of the Gelsolin protein superfamily, like gelsolin itself and villin, modulate apoptosis induction in the gastrointestinal epithelium (Wang et al., 2012; Roy et al., 2018). Villin is a tissue-specific actin-modifying protein (Khurana and George, 2008), which together with gelsolin are targeted to the mitochondria on early steps of the apoptotic pathway (Roy et al., 2018). Their ability to associate with both actin and mitochondria suggest their role in cell survival through the preservation of actin cytoskeleton dynamics in mitochondrial regions controlling the trafficking of anti- and pro-apoptotic signals.

Given the relevance of gelsolin in several aspects of mitochondrial pathophysiology, we will henceforth focus the review on this particular ABP.

\section{GELSOLIN}

Gelsolin (GSN) is an abundant ABP that participates in actinremodeling either by sequestering G-actin or by severing, capping, and nucleating F-actin (Yin and Stossel, 1979; Sun et al., 1999; Feng et al., 2001). This is mediated by calcium concentration, phosphatidylinositol-4,5-bisphosphate $\left(\mathrm{PIP}_{2}\right)$ and $\mathrm{pH}$ (Hu et al., 2016). In human, alternative splicing of GSN mRNA leads to two main isoforms with differentiated functions (Yin et al., 1984): the plasma (pGSN) and cytoplasmic (cGSN) isoforms (UniProtKB reference P06396). Structurally, both isoforms are composed by six gelsolin domains (G1-G6) (Kwiatkowski et al., 1986) divided in two homologous structures: the N-terminal fragment (G1-G3) is involved in actin severing, and the C-terminal fragment (G4G6) coordinates calcium binding (Choe et al., 2002). Domains G3 and G4 are separated by a linker sequence of 70 amino acids that is prone to cleavage by caspase-3 (Kothakota et al., 1997; Kamada et al., 1998).

\section{Plasma GSN}

Secreted pGSN (86 kDa) differs from intracellular cGSN (81 kDa) in its N-terminal sequence, spanning a 51-amino acid secretory peptide, and in the presence of a disulphide bond between cysteine residues 188-201 that enhances its stability in the extracellular media (Kwiatkowski et al., 1988; Wen et al., 
1996). pGSN modulates bacterial immune response, acting as a buffering agent in inflammation (Bucki et al., 2008b; Cheng et al., 2017), and it is a part of the extracellular actin scavenger system (EASS) responsible for rapid severing and clearance of actin filaments released from dead cells into the bloodstream (Lind et al., 1986; Lee and Galbraith, 1992). In conditions of massive cell death, substantial actin release overwhelms the EASS, resulting in a decline of circulating pGSN levels. Consequently, pGSN has been proposed as a biomarker for multiple diseases ( $\mathrm{Li}$ et al., 2012), ranging from cardiovascular pathologies (Khatri et al., 2014; Piktel et al., 2018; Feldt et al., 2019) to major trauma, diabetes, Alzheimer's disease, rheumatoid arthritis, sepsis, liver failure, or cancer, to the point that pGSN has been proposed as a general biomarker of health prognosis (Peddada et al., 2012).

\section{Cytosolic GSN}

Cytosolic GSN (cGSN) is ubiquitously expressed and, besides its main role in actin filament remodeling, it participates in regulatory signaling pathways that require a continuous rearrangement of the actin cytoskeleton, such as the phospholipase C (PLC) or phosphoinositide 3-kinase (PI3K) cascades (Singh et al., 1996; Sun et al., 1997); the epidermal growth factor receptor (EGFR) pathway (Chen et al., 1996; Azuma et al., 1998; De Corte et al., 2002); phagocytosis mediated by the Fc-receptor or integrins (Serrander et al., 2000; Witke et al., 2001; Arora et al., 2004); and also as a transcriptional coactivator of the thyroid (TR) and androgen (AR) receptors (Nishimura et al., 2003; Kim et al., 2007), and of the hypoxia inducible factor (HIF-1) to favor hypoxia-regulated genes expression, GSN itself among them (Greijer et al., 2005; Li Q. et al., 2009). Finally, cGSN also interacts with p53, inhibiting its nucleus translocation (An et al., 2011).

Besides, cGSN is associated to membrane regions of the cell rich in actin filaments such as the ER, vesicles or mitochondrial membranes (Cooper et al., 1988; Hartwig et al., 1989). cGSN co-purifies with isolated mitochondria, where it interacts with the major OMM channel protein VDAC to promote cell survival responses (Figure 1B) (Koya et al., 2000; Kusano et al., 2000; García-Bartolomé et al., 2017). In fact, cGSN is as a dual regulator of apoptotic cell death due to its cleavage by caspase-3 in two independent fragments (Kothakota et al., 1997). The C-terminal fragment, of $41 \mathrm{kDa}$, as well as fulllength cGSN, may act as anti-apoptotic factors through VDAC blockage, thus avoiding Cyt c release into the cytosol (Koya et al., 2000; Kusano et al., 2000). Both can also bind to actin and DNaseI, forming a ternary complex that prevents the nuclear translocation of DNaseI. In contrast, the proapoptotic $\mathrm{N}$-terminal fragment, of $39 \mathrm{kDa}$, may severe actin filaments in a calcium-independent manner (Kothakota et al., 1997; Geng et al., 1998; Kamada et al., 1998). It competes with actin for DNaseI binding, releasing it from the GSN:actin: DNaseI ternary complex and promoting its nuclear translocation, ultimately leading to nuclear DNA degradation and apoptosis (Chhabra et al., 2005; Li Q. et al., 2009). Furthermore, cGSN overexpression may inhibit the apoptotic pathway by sequestering and inactivating caspase 3 in a GSN:PIP2:caspase3 complex (Ohtsu et al.,
1997), and by precluding nuclear translocation of p53 (An et al., 2011). This protective role of cGSN was also demonstrated in mouse models of Alzheimer's disease, where cGSN overexpression prevented the cytotoxic effect induced by accumulation of the amyloid beta $(A \beta)$ peptide on mitochondrial function and cell death (Qiao et al., 2005; Antequera et al., 2009). High levels of cGSN were also reported in experimental models mainly exhibiting oxidative stress, such as upon hydrogen peroxide treatment, (Chauhan et al., 2008; Ji et al., 2010), as a consequence of intracellular calcium alterations (Bucki et al., 2008a), under HIF-1-modulated hypoxia (Nishimura et al., 2003), and in pathophysiological alterations like ageing and senescence (Ahn, 2003), Down syndrome (Ji et al., 2009), and heart failure (Li G. H. et al., 2009; Patel et al., 2018).

\section{GSN and Mitochondrial Disease}

The relative abundance of cGSN also increases in primary fibroblasts from patients and cellular models of OXPHOS system deficiency (Marín-Buera et al., 2015; García-Bartolomé et al., 2017; García-Bartolomé et al., 2020), suggesting its protective role through the regulation of cell survival responses. In these models, there was a reverse correlation between increased cGSN and decreased pGSN levels, resulting in a significantly high cGSN:pGSN protein ratio as a novel hallmark of OXPHOS dysfunction (García-Bartolomé et al., 2020). Interestingly, pGSN levels significantly decreased in OXPHOS-deficient patients, which reinforced the diagnosis accuracy for these disorders of the formerly reported biomarkers GDF-15 and FGF-21 (Peñas et al., 2021). These data suggest a tightly regulated coordination of both GSN isoforms, whose relevance in mitochondrial pathophysiology remains unknown.

\section{CONCLUSIONS AND PERSPECTIVES}

We have emphasized the importance of the actin cytoskeletonmediated regulation on several aspects of mitochondrial (dys) function, and detailed the so-far known role of one of the most abundant ABPs, Gelsolin, in these processes. It remains unknown whether the apparently protective role of GSN, like other ABPs, directly impacts on mitochondrial function or whether it indirectly functions through regulation of actin cytoskeleton dynamics. Either way, the functional interplay between GSN isoforms in health and disease, as well as that between the actin cytoskeleton, $\mathrm{ABPs}$ and mitochondrial membranes for the regulation of cellular homeostasis and metabolism, open new exciting possibilities for future research.

\section{AUTHOR CONTRIBUTIONS}

Conceptualization and Investigation, MI, AP and CU; Writing Original Draft, MI and AP; Writing - Review and Editing, CU; Visualization, MI; Supervision, JA, MAM and CU; Project 
Administration, JA, MAM and CU; Funding Acquisition, MAM and CU.

\section{FUNDING}

Research was funded by Instituto de Salud Carlos IIIMINECO/European FEDER Funds Grants PI17-00048 and

\section{REFERENCES}

Abrisch, R. G., Gumbin, S. C., Wisniewski, B. T., Lackner, L. L., and Voeltz, G. K. (2020). Fission and Fusion Machineries Converge at ER Contact Sites to Regulate Mitochondrial Morphology. J. Cel Biol. 219, e201911122. doi:10.1083/jcb.201911122

Ahn, J. S., Jang, I.-S., Rhim, J. H., Kim, K., Yeo, E.-J., and Park, S. C. (2003). Gelsolin for Senescence-Associated Resistance to Apoptosis. Ann. N. Y. Acad. Sci. 1010, 493-495. doi:10.1196/annals.1299.090

An, J.-H., Kim, J.-W., Jang, S.-M., Kim, C.-H., Kang, E.-J., and Choi, K.-H. (2011). Gelsolin Negatively Regulates the Activity of Tumor Suppressor P53 through Their Physical Interaction in Hepatocarcinoma HepG2 Cells. Biochem. Biophysical Res. Commun. 412, 44-49. doi:10.1016/ j.bbrc.2011.07.034

Antequera, D., Vargas, T., Ugalde, C., Spuch, C., Molina, J. A., Ferrer, I., et al. (2009). Cytoplasmic Gelsolin Increases Mitochondrial Activity and Reduces A $\beta$ burden in a Mouse Model of Alzheimer's Disease. Neurobiol. Dis. 36, 42-50. doi:10.1016/j.nbd.2009.06.018

Arnold, H., and Pette, D. (1968). Binding of Glycolytic Enzymes to Structure Proteins of the Muscle. Eur. J. Biochem. 6, 163-171. doi:10.1111/j.14321033.1968.tb00434.x

Arora, P. D., Glogauer, M., Kapus, A., Kwiatkowski, D. J., and McCulloch, C. A. (2004). Gelsolin Mediates Collagen Phagocytosis through a Rac-dependent Step. $M B o C$ 15, 588-599. doi:10.1091/mbc.e03-07-0468

Azuma, T., Witke, W., Stossel, T. P., Hartwig, J. H., and Kwiatkowski, D. J. (1998). Gelsolin Is a Downstream Effector of Rac for Fibroblast Motility. EMBO J. 17, 1362-1370. doi:10.1093/emboj/17.5.1362

Birsa, N., Norkett, R., Wauer, T., Mevissen, T. E. T., Wu, H.-C., Foltynie, T., et al. (2014). Lysine 27 Ubiquitination of the Mitochondrial Transport Protein Miro Is Dependent on Serine 65 of the Parkin Ubiquitin Ligase. J. Biol. Chem. 289, 14569-14582. doi:10.1074/jbc.M114.563031

Boldogh, I. R., and Pon, L. A. (2006). Interactions of Mitochondria with the Actin Cytoskeleton. Biochim. Biophys. Acta (Bba) - Mol. Cel Res. 1763, 450-462. doi:10.1016/j.bbamcr.2006.02.014

Bononi, A., Missiroli, S., Poletti, F., Suski, J. M., Agnoletto, C., Bonora, M., et al. (2012). Mitochondria-Associated Membranes (MAMs) as Hotspot Ca2+ Signaling Units. Adv. Exp. Med. Biol. 740, 411-437. doi:10.1007/978-94-0072888-2_17

Brookes, P. S., Yoon, Y., Robotham, J. L., Anders, M. W., and Sheu, S.-S. (2004). Calcium, ATP, and ROS: a Mitochondrial Love-Hate triangle. Am. J. Physiology-Cell Physiol. 287, C817-C833. doi:10.1152/ajpcell.00139.2004

Bucki, R., Byfield, F. J., Kulakowska, A., McCormick, M. E., Drozdowski, W., Namiot, Z., et al. (2008a). Extracellular Gelsolin Binds Lipoteichoic Acid and Modulates Cellular Response to Proinflammatory Bacterial Wall Components. J. Immunol. 181, 4936-4944. doi:10.4049/ jimmunol.181.7.4936

Bucki, R., Levental, I., Kulakowska, A., and Janmey, P. (2008b). Plasma Gelsolin: Function, Prognostic Value, and Potential Therapeutic Use. Cpps 9, 541-551. doi:10.2174/138920308786733912

Chakrabarti, R., Ji, W.-K., Stan, R. V., Sanz, J., de Juan Sanz, T. A., and Higgs, H. N. (2018). INF2-mediated Actin Polymerization at the ER Stimulates Mitochondrial Calcium Uptake, Inner Membrane Constriction, and Division. J. Cel Biol. 217, 251-268. doi:10.1083/jcb.201709111

Chan, D. C. (2020). Mitochondrial Dynamics and its Involvement in Disease. Annu. Rev. Pathol. Mech. Dis. 15, 235-259. doi:10.1146/annurev-pathmechdis012419-032711
PI20-00057 (to CU) and PI18-01374 (to MAM), and by Comunidad Autónoma de Madrid/ERDF-ESF Grant P2018/ BAA-4403 (to CU).

\section{ACKNOWLEDGMENTS}

We thank all lab members for constructive discussions.

Chauhan, V., Ji, L., and Chauhan, A. (2008). Anti-amyloidogenic, Anti-oxidant and Anti-apoptotic Role of Gelsolin in Alzheimer's Disease. Biogerontology 9, 381-389. doi:10.1007/s10522-008-9169-z

Chen, P., Murphy-Ullrich, J. E., and Wells, A. (1996). A Role for Gelsolin in Actuating Epidermal Growth Factor Receptor-Mediated Cell Motility. J. Cel Biol. 134, 689-698. doi:10.1083/jcb.134.3.689

Cheng, Y., Hu, X., Liu, C., Chen, M., Wang, J., Wang, M., et al. (2017). Gelsolin Inhibits the Inflammatory Process Induced by LPS. Cell. Physiol. Biochem. 41, 205-212. doi:10.1159/000456043

Chhabra, D., Nosworthy, N. J., and Dos Remedios, C. G. (2005). TheN-terminal Fragment of Gelsolin Inhibits the Interaction of DNase I with Isolated Actin, but Not with the Cofilin-Actin Complex. Proteomics 5, 3131-3136. doi:10.1002/ pmic. 200401127

Choe, H., Burtnick, L. D., Mejillano, M., Yin, H. L., Robinson, R. C., and Choe, S. (2002). The Calcium Activation of Gelsolin: Insights from the $3 \AA$ Structure of the G4-G6/Actin Complex. J. Mol. Biol. 324, 691-702. doi:10.1016/S00222836(02)01131-2

Chua, B. T., Volbracht, C., Tan, K. O., Li, R., Yu, V. C., and Li, P. (2003). Mitochondrial Translocation of Cofilin Is an Early Step in Apoptosis Induction. Nat. Cel Biol. 5, 1083-1089. doi:10.1038/ncb1070

Clayton, D. A. (1991). Replication and Transcription of Vertebrate Mitochondrial DNA. Annu. Rev. Cel. Biol. 7, 453-478. doi:10.1146/ annurev.cb.07.110191.002321

Cooper, J. A., Loftus, D. J., Frieden, C., Bryan, J., and Elson, E. L. (1988). Localization and Mobility of Gelsolin in Cells. J. Cel Biol. 106, 1229-1240. doi:10.1083/jcb.106.4.1229

Dadsena, S., King, L. E., and García-Sáez, A. J. (2021). Apoptosis Regulation at the Mitochondria Membrane Level. Biochim. Biophys. Acta (Bba) - Biomembranes 1863, 183716-10. doi:10.1016/j.bbamem.2021.183716

De Corte, V., Bruyneel, E., Boucherie, C., Mareel, M., Vandekerckhove, J., and Gettemans, J. (2002). Gelsolin-induced Epithelial Cell Invasion Is Dependent on Ras-Rac Signaling. EMBO J. 21, 6781-6790. doi:10.1093/emboj/cdf680

De Vos, K. J., Allan, V. J., Grierson, A. J., and Sheetz, M. P. (2005). Mitochondrial Function and Actin Regulate Dynamin-Related Protein 1-dependent Mitochondrial Fission. Curr. Biol. 15, 678-683. doi:10.1016/j.cub.2005.02.064

Feldt, J., Schicht, M., Garreis, F., Welss, J., Schneider, U. W., and Paulsen, F. (2019). Structure, Regulation and Related Diseases of the Actin-Binding Protein Gelsolin. Expert Rev. Mol. Med. 20, e7. doi:10.1017/erm.2018.7

Feng, L., Mejillano, M., Yin, H. L., Chen, J., and Prestwich, G. D. (2001). Fullcontact Domain Labeling: Identification of a Novel Phosphoinositide Binding Site on Gelsolin that Requires the Complete Protein. Biochemistry 40, 904-913. doi:10.1021/bi000996q

Fernie, A. R., Zhang, Y., and Sampathkumar, A. (2020). Cytoskeleton Architecture Regulates Glycolysis Coupling Cellular Metabolism to Mechanical Cues. Trends Biochem. Sci. 45, 637-638. doi:10.1016/j.tibs.2020.04.003

Francy, C. A., Alvarez, F. J. D., Zhou, L., Ramachandran, R., and Mears, J. A. (2015). The Mechanoenzymatic Core of Dynamin-Related Protein 1 Comprises the Minimal Machinery Required for Membrane Constriction. J. Biol. Chem. 290, 11692-11703. doi:10.1074/jbc.M114.610881

Franklin-Tong, V. E., and Gourlay, C. W. (2008). A Role for Actin in Regulating Apoptosis/programmed Cell Death: Evidence Spanning Yeast, Plants and Animals. Biochem. J. 413, 389-404. doi:10.1042/BJ20080320

Friedman, J. R., Lackner, L. L., West, M., DiBenedetto, J. R., Nunnari, J., and Voeltz, G. K. (2011). ER Tubules Mark Sites of Mitochondrial Division. Science 334, 358-362. doi:10.1126/science. 1207385

García-Bartolomé, A., Peñas, A., Illescas, M., Bermejo, V., López-Calcerrada, S., Pérez-Pérez, R., et al. (2020). Altered Expression Ratio of Actin-Binding 
Gelsolin Isoforms Is a Novel Hallmark of Mitochondrial OXPHOS Dysfunction. Cells 9, 1922-21. doi:10.3390/cells9091922

García-Bartolomé, A., Peñas, A., Marín-Buera, L., Lobo-Jarne, T., Pérez-Pérez, R., Morán, M., et al. (2017). Respiratory Chain Enzyme Deficiency Induces Mitochondrial Location of Actin-Binding Gelsolin to Modulate the Oligomerization of VDAC Complexes and Cell Survival. Hum. Mol. Genet. 26, 2493-2506. doi:10.1093/hmg/ddx144

Geng, Y.-J., Azuma, T., Tang, J. X., Hartwig, J. H., Muszynski, M., Wu, Q., et al. (1998). Caspase-3-induced Gelsolin Fragmentation Contributes to Actin Cytoskeletal Collapse, Nucleolysis, and Apoptosis of Vascular Smooth Muscle Cells Exposed to Proinflammatory Cytokines. Eur. J. Cel Biol. 77, 294-302. doi:10.1016/S0171-9335(98)80088-5

Gourlay, C. W., and Ayscough, K. R. (2005). The Actin Cytoskeleton: A Key Regulator of Apoptosis and Ageing? Nat. Rev. Mol. Cel Biol. 6, 583-589. doi:10.1038/nrm1682

Greijer, A., van der Groep, P., Kemming, D., Shvarts, A., Semenza, G., Meijer, G., et al. (2005). Up-regulation of Gene Expression by Hypoxia Is Mediated Predominantly by Hypoxia-Inducible Factor 1 (HIF-1). J. Pathol. 206, 291-304. doi:10.1002/path.1778

Guo, Y., Li, D., Zhang, S., Yang, Y., Liu, J.-J., Wang, X., et al. (2018). Visualizing Intracellular Organelle and Cytoskeletal Interactions at Nanoscale Resolution on Millisecond Timescales. Cell 175, 1430-1442. doi:10.1016/ j.cell.2018.09.057

Han, S., Zhao, F., Hsia, J., Ma, X., Liu, Y., Torres, S., et al. (2021). The Role of Mfn2 in the Structure and Function of Endoplasmic Reticulum-Mitochondrial Tethering In Vivo. J. Cel Sci. 134, jcs253443. doi:10.1242/jcs.253443

Hartwig, J. H., Chambers, K. A., and Stossel, T. P. (1989). Association of Gelsolin with Actin Filaments and Cell Membranes of Macrophages and Platelets. J. Cel Biol. 108, 467-479. doi:10.1083/jcb.108.2.467

Hatch, A. L., Gurel, P. S., and Higgs, H. N. (2014). Novel Roles for Actin in Mitochondrial Fission. J. Cel Sci. 127, 4549-4560. doi:10.1242/jcs.153791

Hirokawa, N., and Takemura, R. (2005). Molecular Motors and Mechanisms of Directional Transport in Neurons. Nat. Rev. Neurosci. 6, 201-214. doi:10.1038/ nrn1624

Hu, H., Juvekar, A., Lyssiotis, C. A., Lien, E. C., Albeck, J. G., Oh, D., et al. (2016). Phosphoinositide 3-Kinase Regulates Glycolysis through Mobilization of Aldolase from the Actin Cytoskeleton. Cell 164, 433-446. doi:10.1016/ j.cell.2015.12.042

Hu, J., Zhang, H., Li, J., Jiang, X., Zhang, Y., Wu, Q., et al. (2020). ROCK1 Activation-Mediated Mitochondrial Translocation of Drp1 and Cofilin Are Required for Arnidiol-Induced Mitochondrial Fission and Apoptosis. J. Exp. Clin. Cancer Res. 39, 1-16. doi:10.1186/s13046-02001545-7

Ji, L., Chauhan, A., and Chauhan, V. (2010). Upregulation of Cytoplasmic Gelsolin, an Amyloid- $\beta$-Binding Protein, under Oxidative Stress Conditions: Involvement of Protein Kinase C. Jad 19, 829-838. doi:10.3233/JAD-2010-1281

Ji, L., Chauhan, A., Muthaiyah, B., Wegiel, J., and Chauhan, V. (2009). Gelsolin Levels Are Increased in the Brain as a Function of Age During Normal Development in Children that Are Further Increased in Down Syndrome. Alzheimer Dis. Assoc. Disord. 23, 319-322. doi:10.1097/ WAD.0b013e31819d494e

Ji, W.-k., Hatch, A. L., Merrill, R. A., Strack, S., and Higgs, H. N. (2015). Actin Filaments Target the Oligomeric Maturation of the Dynamin GTPase Drp1 to Mitochondrial Fission Sites. Elife 4, 1-25. doi:10.7554/eLife.11553

Kamada, S., Kusano, H., Fujita, H., Ohtsu, M., Koya, R. C., Kuzumaki, N., et al. (1998). A Cloning Method for Caspase Substrates that Uses the Yeast TwoHybrid System: Cloning of the Antiapoptotic Gene Gelsolin. Proc. Natl. Acad. Sci. 95, 8532-8537. doi:10.1073/pnas.95.15.8532

Kast, D. J., and Dominguez, R. (2017). The Cytoskeleton-Autophagy Connection. Curr. Biol. 27, R318-R326. doi:10.1016/j.cub.2017.02.061

Kayalar, C., Örd, T., Testa, M. P., Zhong, L. T., and Bredesen, D. E. (1996). Cleavage of Actin by Interleukin 1 Beta-Converting Enzyme to Reverse DNase I Inhibition. Proc. Natl. Acad. Sci. 93, 2234-2238. doi:10.1073/ pnas.93.5.2234

Khatri, N., Sagar, A., Peddada, N., Choudhary, V., Chopra, B. S., Garg, V., et al. (2014). Plasma Gelsolin Levels Decrease in Diabetic State and Increase upon Treatment with F-Actin Depolymerizing Versions of Gelsolin. J. Diabetes Res. 2014, 1-8. doi:10.1155/2014/152075
Khurana, S., and George, S. P. (2008). Regulation of Cell Structure and Function by Actin-Binding Proteins: Villin's Perspective. FEBS Lett. 582, 2128-2139. doi:10.1016/j.febslet.2008.02.040

Kim, C. S., Furuya, F., Ying, H., Kato, Y., Hanover, J. A., and Cheng, S.-y. (2007). Gelsolin: A Novel Thyroid Hormone Receptor- $\beta$ Interacting Protein that Modulates Tumor Progression in a Mouse Model of Follicular Thyroid Cancer. Endocrinology 148, 1306-1312. doi:10.1210/en.2006-0923

Klamt, F., Zdanov, S., Levine, R. L., Pariser, A., Zhang, Y., Zhang, B., et al. (2009). Oxidant-induced Apoptosis Is Mediated by Oxidation of the Actin-Regulatory Protein Cofilin. Nat. Cel Biol 11, 1241-1246. doi:10.1038/ncb1968

Kornmann, B., and Walter, P. (2010). ERMES-mediated ER-Mitochondria Contacts: Molecular Hubs for the Regulation of Mitochondrial Biology. J. Cel Sci. 123, 1389-1393. doi:10.1242/jcs.058636

Korobova, F., Gauvin, T. J., and Higgs, H. N. (2014). A Role for Myosin II in Mammalian Mitochondrial Fission. Curr. Biol. 24, 409-414. doi:10.1016/ j.cub.2013.12.032

Korobova, F., Ramabhadran, V., and Higgs, H. N. (2013). An Actin-Dependent Step in Mitochondrial Fission Mediated by the ER-Associated Formin INF2. Science 339, 464-467. doi:10.1126/science.1228360

Kothakota, S., Azuma, T., Reinhard, C., Klippel, A., Tang, J., Chu, K., et al. (1997). Caspase-3-generated Fragment of Gelsolin: Effector of Morphological Change in Apoptosis. Science 278, 294-298. doi:10.1126/science.278.5336.294

Koya, R. C., Fujita, H., Shimizu, S., Ohtsu, M., Takimoto, M., Tsujimoto, Y., et al. (2000). Gelsolin Inhibits Apoptosis by Blocking Mitochondrial Membrane Potential Loss and Cytochrome C Release. J. Biol. Chem. 275, 15343-15349. doi:10.1074/jbc.275.20.15343

Kruppa, A. J., and Buss, F. (2021). Motor Proteins at the MitochondriaCytoskeleton Interface. J. Cel Sci. 134, jcs226084. doi:10.1242/jcs.226084

Kusano, H., Shimizu, S., Koya, R. C., Fujita, H., Kamada, S., Kuzumaki, N., et al. (2000). Human Gelsolin Prevents Apoptosis by Inhibiting Apoptotic Mitochondrial Changes via Closing VDAC. Oncogene 19, 4807-4814. doi:10.1038/sj.onc.1203868

Kwiatkowski, D. J., Stossel, T. P., Orkin, S. H., Mole, J. E., Coltens, H. R., and Yin, H. L. (1986). Plasma and Cytoplasmic Gelsolins Are Encoded by a Single Gene and Contain a Duplicated Actin-Binding Domain. Nature 323, 455-458. doi:10.1038/323455a0

Kwiatkowski, D., Mehl, R., and Yin, H. (1988). Genomic Organization and Biosynthesis of Secreted and Cytoplasmic Forms of Gelsolin. J. Cel Biol. 106, 375-384. doi:10.1083/jcb.106.2.375

Lapeña-Luzón, T., Rodríguez, L. R., Beltran-Beltran, V., Benetó, N., Pallardó, F. V., and Gonzalez-Cabo, P. (2021). Cofilin and Neurodegeneration: New Functions for an Old but Gold Protein. Brain Sci. 11, 954. doi:10.3390/ brainsci1 1070954

Lappalainen, P. (2016). Actin-binding Proteins: the Long Road to Understanding the Dynamic Landscape of Cellular Actin Networks. MBoC 27, 2519-2522. doi:10.1091/mbc.e15-10-0728

Lee, W. M., and Galbraith, R. M. (1992). The Extracellular Actin-Scavenger System and Actin Toxicity. N. Engl. J. Med. 326, 1335-1341. doi:10.1056/ NEJM199205143262006

Lewis, S. C., Uchiyama, L. F., and Nunnari, J. (2016). ER-mitochondria Contacts Couple mtDNA Synthesis with Mitochondrial Division in Human Cells. Science 353, aaf5549. doi:10.1126/science.aaf5549

Li, G. H., Arora, P. D., Chen, Y., McCulloch, C. A., and Liu, P. (2012). Multifunctional Roles of Gelsolin in Health and Diseases. Med. Res. Rev. 32, 999-1025. doi:10.1002/med.20231

Li, G. H., Shi, Y., Chen, Y., Sun, M., Sader, S., Maekawa, Y., et al. (2009a). Gelsolin Regulates Cardiac Remodeling after Myocardial Infarction through DNase I-Mediated Apoptosis. Circ. Res. 104, 896-904. doi:10.1161/ CIRCRESAHA.108.172882

Li, Q., Ye, Z., Wen, J., Ma, L., He, Y., Lian, G., et al. (2009b). Gelsolin, but Not its Cleavage, Is Required for TNF-Induced ROS Generation and Apoptosis in MCF-7 Cells. Biochem. Biophysical Res. Commun. 385, 284-289. doi:10.1016/ j.bbrc.2009.05.078

Lind, S. E., Smith, D. B., Janmey, P. A., and Stossel, T. P. (1986). Role of Plasma Gelsolin and the Vitamin D-Binding Protein in Clearing Actin from the Circulation. J. Clin. Invest. 78, 736-742. doi:10.1172/JCI112634

López-Doménech, G., Covill-Cooke, C., Ivankovic, D., Halff, E. F., Sheehan, D. F., Norkett, R., et al. (2018). Miro Proteins Coordinate Microtubule- and Actin- 
dependent Mitochondrial Transport and Distribution. EMBO J. 37, 321-336. doi:10.15252/embj.201696380

Man, S. M., and Kanneganti, T.-D. (2016). Converging Roles of Caspases in Inflammasome Activation, Cell Death and Innate Immunity. Nat. Rev. Immunol. 16, 7-21. doi:10.1038/nri.2015.7

Manor, U., Bartholomew, S., Golani, G., Christenson, E., Kozlov, M., Higgs, H., et al. (2015). A Mitochondria-Anchored Isoform of the Actin-Nucleating Spire Protein Regulates Mitochondrial Division. Elife 4, 1-27. doi:10.7554/ eLife. 08828

Marín-Buera, L., García-Bartolomé, A., Morán, M., López-Bernardo, E., Cadenas, S., Hidalgo, B., et al. (2015). Differential Proteomic Profiling Unveils New Molecular Mechanisms Associated with Mitochondrial Complex III Deficiency. J. Proteomics 113, 38-56. doi:10.1016/j.jprot.2014.09.007

Merino, F., Pospich, S., and Raunser, S. (2020). Towards a Structural Understanding of the Remodeling of the Actin Cytoskeleton. Semin. Cel Dev. Biol. 102, 51-64. doi:10.1016/j.semcdb.2019.11.018

Moore, A. S., Wong, Y. C., Simpson, C. L., and Holzbaur, E. L. F. (2016). Dynamic Actin Cycling through Mitochondrial Subpopulations Locally Regulates the Fission-Fusion Balance within Mitochondrial Networks. Nat. Commun. 7, 12886. doi:10.1038/ncomms 12886

Ni, H.-M., Williams, J. A., and Ding, W.-X. (2015). Mitochondrial Dynamics and Mitochondrial Quality Control. Redox Biol. 4, 6-13. doi:10.1016/ j.redox.2014.11.006

Nishimura, K., Ting, H. J., Harada, Y., Tokizane, T., Nonomura, N., Kang, H. Y., et al. (2003). Modulation of Androgen Receptor Transactivation by Gelsolin: a Newly Identified Androgen Receptor Coregulator. Cancer Res. 63, 4888-4894. PMID: 12941811.

Nunnari, J., and Suomalainen, A. (2012). Mitochondria: In Sickness and in Health. Cell 148, 1145-1159. doi:10.1016/j.cell.2012.02.035

Odaka, C., Sanders, M. L., and Crews, P. (2000). Jasplakinolide Induces Apoptosis in Various Transformed Cell Lines by a Caspase-3-like Protease-dependent Pathway. Clin. Diagn. Lab. Immunol. 7, 947-952. doi:10.1128/CDLI.7.6.947952.2000

Ohtsu, M., Sakai, N., Fujita, H., Kashiwagi, M., Gasa, S., Shimizu, S., et al. (1997). Inhibition of Apoptosis by the Actin-Regulatory Protein Gelsolin. EMBO J. 16, 4650-4656. doi:10.1093/emboj/16.15.4650

Pagliuso, A., Tham, T. N., Stevens, J. K., Lagache, T., Persson, R., Salles, A., et al. (2016). A Role for Septin 2 in Drp1-mediated Mitochondrial Fission. EMBO Rep. 17, 858-873. doi:10.15252/embr.201541612

Park, J. S., Burckhardt, C. J., Lazcano, R., Solis, L. M., Isogai, T., Li, L., et al. (2020). Mechanical Regulation of Glycolysis via Cytoskeleton Architecture. Nature 578, 621-626. doi:10.1038/s41586-020-1998-1

Patel, V. B., Zhabyeyev, P., Chen, X., Wang, F., Paul, M., Fan, D., et al. (2018). PI3Ka-regulated Gelsolin Activity Is a Critical Determinant of Cardiac Cytoskeletal Remodeling and Heart Disease. Nat. Commun. 9, 5390. doi:10.1038/s41467-018-07812-8

Peddada, N., Sagar, A., Ashishand Garg, R. (2012). Plasma Gelsolin: A General Prognostic Marker of Health. Med. Hypotheses 78, 203-210. doi:10.1016/ j.mehy.2011.10.024

Peñas, A., Fernández-De la Torre, M., Laine-Menéndez, S., Lora, D., Illescas, M., García-Bartolomé, A., et al. (2021). Plasma Gelsolin Reinforces the Diagnostic Value of FGF-21 and GDF-15 for Mitochondrial Disorders. Ijms 22, 6396. doi:10.3390/ijms22126396

Piktel, E., Levental, I., Durnaś, B., Janmey, P., and Bucki, R. (2018). Plasma Gelsolin: Indicator of Inflammation and its Potential as a Diagnostic Tool and Therapeutic Target. Ijms 19, 2516. doi:10.3390/ijms19092516

Portt, L., Norman, G., Clapp, C., Greenwood, M., and Greenwood, M. T. (2011). Anti-apoptosis and Cell Survival: A Review. Biochim. Biophys. Acta (Bba) - Mol. Cel Res. 1813, 238-259. doi:10.1016/j.bbamcr.2010.10.010

Qiao, H., Koya, R. C., Nakagawa, K., Tanaka, H., Fujita, H., Takimoto, M., et al. (2005). Inhibition of Alzheimer's Amyloid- $\beta$ Peptide-Induced Reduction of Mitochondrial Membrane Potential and Neurotoxicity by Gelsolin. Neurobiol. Aging 26, 849-855. doi:10.1016/j.neurobiolaging.2004.08.003

Rampelt, H., and Pfanner, N. (2016). Coordination of Two Genomes by Mitochondrial Translational Plasticity. Cell 167, 308-310. doi:10.1016/ j.cell.2016.09.042

Rehklau, K., Gurniak, C. B., Conrad, M., Friauf, E., Ott, M., and Rust, M. B. (2012). ADF/cofilin Proteins Translocate to Mitochondria during Apoptosis but Are
Not Generally Required for Cell Death Signaling. Cell Death Differ 19, 958-967. doi:10.1038/cdd.2011.180

Rehklau, K., Hoffmann, L., Gurniak, C. B., Ott, M., Witke, W., Scorrano, L., et al. (2017). Cofilin1-dependent Actin Dynamics Control DRP1-Mediated Mitochondrial Fission. Cell Death Dis 8, e3063. doi:10.1038/cddis.2017.448

Reid, R. A., Moyle, J., and Mitchell, P. (1966). Synthesis of Adenosine Triphosphate by a Protonmotive Force in Rat Liver Mitochondria. Nature 212, 257-258. doi:10.1038/212257a0

Reyes, A., He, J., Mao, C. C., Bailey, L. J., Di Re, M., Sembongi, H., et al. (2011). Actin and Myosin Contribute to Mammalian Mitochondrial DNA Maintenance. Nucleic Acids Res. 39, 5098-5108. doi:10.1093/nar/gkr052

Rintoul, G. L., Filiano, A. J., Brocard, J. B., Kress, G. J., and Reynolds, I. J. (2003). Glutamate Decreases Mitochondrial Size and Movement in Primary Forebrain Neurons. J. Neurosci. 23, 7881-7888. doi:10.1523/jneurosci.2321-07881.2003

Roh, S. E., Woo, J. A., Lakshmana, M. K., Uhlar, C., Ankala, V., Boggess, T., et al. (2013). Mitochondrial Dysfunction and Calcium Deregulation by the RanBP9cofilin Pathway. FASEB j. 27, 4776-4789. doi:10.1096/fj.13-234765

Rohn, J. L., Patel, J. V., Neumann, B., Bulkescher, J., McHedlishvili, N., McMullan, R. C., et al. (2014). Myo19 Ensures Symmetric Partitioning of Mitochondria and Coupling of Mitochondrial Segregation to Cell Division. Curr. Biol. 24, 2598-2605. doi:10.1016/j.cub.2014.09.045

Roman, I., Figys, J., Steurs, G., and Zizi, M. (2006). Direct Measurement of VDACActin Interaction by Surface Plasmon Resonance. Biochim. Biophys. Acta (Bba) - Biomembranes 1758, 479-486. doi:10.1016/j.bbamem.2006.03.019

Roy, S., Esmaeilniakooshkghazi, A., Patnaik, S., Wang, Y., George, S. P., Ahrorov, A., et al. (2018). Villin-1 and Gelsolin Regulate Changes in Actin Dynamics that Affect Cell Survival Signaling Pathways and Intestinal Inflammation. Gastroenterology 154, 1405-1420. doi:10.1053/j.gastro.2017.12.016

Schwarz, T. L. (2013). Mitochondrial Trafficking in Neurons. Cold Spring Harbor Perspect. Biol. 5, a011304. doi:10.1101/cshperspect.a011304

Scott, I., and Youle, R. J. (2010). Mitochondrial Fission and Fusion. Essays Biochem. 47, 85-98. doi:10.1042/bse 0470085

Serrander, L., Skarman, P., Rasmussen, B., Witke, W., Lew, D. P., Krause, K.-H., et al. (2000). Selective Inhibition of IgG-Mediated Phagocytosis in GelsolinDeficient Murine Neutrophils. J. Immunol. 165, 2451-2457. doi:10.4049/ jimmunol.165.5.2451

Sheng, Z.-H. (2014). Mitochondrial Trafficking and Anchoring in Neurons: New Insight and Implications. J. Cel Biol. 204, 1087-1098. doi:10.1083/ jcb. 201312123

Shneyer, B. I., Usaj, M., and Henn, A. (2016). Myo19 Is an Outer Mitochondrial Membrane Motor and Effector of Starvation Induced Filopodia, J. Cell Sci. , 543-556. doi:10.1242/jcs.175349

Silacci, P., Mazzolai, L., Gauci, C., Stergiopulos, N., Yin, H. L., and Hayoz, D. (2004). Gelsolin Superfamily Proteins: Key Regulators of Cellular Functions. CMLS, Cell. Mol. Life Sci. 61, 2614-2623. doi:10.1007/s00018-004-4225-6

Singh, S. S., Chauhan, A., Murakami, N., and Chauhan, V. P. S. (1996). Profilin and Gelsolin Stimulate Phosphatidylinositol 3-kinase Activity. Biochemistry 35, 16544-16549. doi:10.1021/bi9609634

Spelbrink, J. N. (2009). Functional Organization of Mammalian Mitochondrial DNA in Nucleoids: History, Recent Developments, and Future Challenges. IUBMB Life 62, a-n. doi:10.1002/iub.282

Sun, H.-q., Lin, K.-m., and Yin, H. L. (1997). Gelsolin Modulates Phospholipase C Activity In Vivo through Phospholipid Binding. J. Cel Biol. 138, 811-820. doi:10.1083/jcb.138.4.811

Sun, H. Q., Yamamoto, M., Mejillano, M., Yin, H. L., Yamamoto, M., Mejillano, M., et al. (1999). Gelsolin, a Multifunctional Actin Regulatory Protein. J. Biol. Chem. 274, 33179-33182. doi:10.1074/jbc.274.47.33179

Takahashi, K., Miura, Y., Ohsawa, I., Shirasawa, T., and Takahashi, M. (2018). In Vitro rejuvenation of Brain Mitochondria by the Inhibition of Actin Polymerization. Sci. Rep. 8, 2-11. doi:10.1038/s41598-018-34006-5

Tilokani, L., Nagashima, S., Paupe, V., and Prudent, J. (2018). Mitochondrial Dynamics: Overview of Molecular Mechanisms. Essays Biochem. 62, 341-360. doi:10.1042/EBC20170104

Utsumi, T., Sakurai, N., Nakano, K., and Ishisaka, R. (2003). C-terminal 15 kDa Fragment of Cytoskeletal Actin Is posttranslationallyN-Myristoylated upon Caspase-Mediated Cleavage and Targeted to Mitochondria. FEBS Lett. 539, 37-44. doi:10.1016/S0014-5793(03)00180-7 
van Spronsen, M., Mikhaylova, M., Lipka, J., Schlager, M. A., van den Heuvel, D. J., Kuijpers, M., et al. (2013). TRAK/Milton Motor-Adaptor Proteins Steer Mitochondrial Trafficking to Axons and Dendrites. Neuron 77, 485-502. doi:10.1016/j.neuron.2012.11.027

Venit, T., El Said, N. H., Mahmood, S. R., and Percipalle, P. (2021). A Dynamic Actin-dependent Nucleoskeleton and Cell Identity. J. Biochem. 169, 243-257. doi:10.1093/jb/mvaa133

Verburg, J., and Hollenbeck, P. J. (2008). Mitochondrial Membrane Potential in Axons Increases with Local Nerve Growth Factor or Semaphorin Signaling. J. Neurosci. 28, 8306-8315. doi:10.1523/JNEUROSCI.2614-08.2008

Wang, X., Winter, D., Ashrafi, G., Schlehe, J., Wong, Y. L., Selkoe, D., et al. (2011). PINK1 and Parkin Target Miro for Phosphorylation and Degradation to Arrest Mitochondrial Motility. Cell 147, 893-906. doi:10.1016/j.cell.2011.10.018

Wang, Y., George, S. P., Srinivasan, K., Patnaik, S., and Khurana, S. (2012). Actin Reorganization as the Molecular Basis for the Regulation of Apoptosis in Gastrointestinal Epithelial Cells. Cel Death Differ 19, 1514-1524. doi:10.1038/ cdd.2012.28

Wen, D., Corina, K., Chow, E. P., Miller, S., Janmey, P. A., and Pepinsky, R. B. (1996). The Plasma and Cytoplasmic Forms of Human Gelsolin Differ in Disulfide Structure. Biochemistry 35, 9700-9709. doi:10.1021/bi960920n

Witke, W., Li, W., Kwiatkowski, D. J., and Southwick, F. S. (2001). Comparisons of CapG and Gelsolin-Null Macrophages. J. Cel Biol. 154, 775-784. doi:10.1083/ jcb.200101113

Xie, X., Venit, T., Drou, N., and Percipalle, P. (2018). In Mitochondria $\beta$-Actin Regulates mtDNA Transcription and Is Required for Mitochondrial Quality Control. iScience, 3, 226-237. doi:10.1016/j.isci.2018.04.021

Xu, L., Wang, X., and Tong, C. (2020). Endoplasmic Reticulum-Mitochondria Contact Sites and Neurodegeneration. Front. Cel Dev. Biol. 8, 428. doi:10.3389/ fcell.2020.00428

$\mathrm{Xu}$, X., Forbes, J. G., and Colombini, M. (2001). Actin Modulates the Gating of Neurospora Crassa VDAC. J. Membr. Biol. 180, 73-81. doi:10.1007/ s002320010060
Yamazaki, Y., Tsuruga, M., Zhou, D., Fujita, Y., Shang, X., Dang, Y., et al. (2000) Cytoskeletal Disruption Accelerates Caspase-3 Activation and Alters the Intracellular Membrane Reorganization in DNA Damage-Induced Apoptosis. Exp. Cel Res. 259, 64-78. doi:10.1006/excr.2000.4970

Yin, H. L., Kwiatkowski, D. J., Mole, J. E., and Cole, F. S. (1984). Structure and Biosynthesis of Cytoplasmic and Secreted Variants of Gelsolin. J. Biol. Chem. 259, 5271-5276. doi:10.1016/s0021-9258(17)42985-1

Yin, H. L., and Stossel, T. P. (1979). Control of Cytoplasmic Actin Gel-Sol Transformation by Gelsolin, a Calcium-dependent Regulatory Protein. Nature 281, 583-586. doi:10.1038/281583a0

Youle, R. J., and Van Der Bliek, A. M. (2012). Mitochondrial Fission, Fusion, and Stress. Science 337, 1062-1065. doi:10.1126/science.1219855

Conflict of Interest: The authors declare that the research was conducted in the absence of any commercial or financial relationships that could be construed as a potential conflict of interest.

The reviewer EC declared a shared affiliation with the authors to the handling Editor.

Publisher's Note: All claims expressed in this article are solely those of the authors and do not necessarily represent those of their affiliated organizations, or those of the publisher, the editors and the reviewers. Any product that may be evaluated in this article, or claim that may be made by its manufacturer, is not guaranteed or endorsed by the publisher.

Copyright (C) 2021 Illescas, Peñas, Arenas, Martín and Ugalde. This is an open-access article distributed under the terms of the Creative Commons Attribution License (CC $B Y)$. The use, distribution or reproduction in other forums is permitted, provided the original author(s) and the copyright owner(s) are credited and that the original publication in this journal is cited, in accordance with accepted academic practice. No use, distribution or reproduction is permitted which does not comply with these terms. 